\title{
Samasy: an automated system for sample selection and robotic transfer
}

$\overline{\text { Clinton L Cario' \& John S Witte }{ }^{\star, 1,2}}$

\section{ABSTRACT}

Sample automation and management is increasingly important as the number and size of populationscale and high-throughput projects grow. This is particularly the case in large-scale population studies where sample size is far outpacing the commonly used 96-well-plate format. To facilitate management and transfer of samples in this format, we present Samasy, a web-based application for the construction of a sample database, intuitive display of sample and batch information, and facilitation of automated sample transfer or subset. Samasy is designed with ease-of-use in mind, can be quickly set up, and runs in any web browser.

\section{METHOD SUMMARY}

Samasy is a web application developed with Ruby, Sinatra, Javascript, CSS3 and HTML5. It features an interface for the import of 96-well plate sample information into a SQLite3 database, a sample/batch visualization system and the ability to view sample attribute distributions, and can generate sample transfer files for robotic transfer (e.g., Beckman Biomek).

\section{KEYWORDS:}

96-well plate $\cdot$ large-scale genotyping - sample management - sample transfer $\cdot$ web interface

'Department of Epidemiology \& Biostatistics, University of California, San Francisco, CA, USA; ${ }^{2}$ Institute for Human Genetics, University of California, San Francisco, CA, USA; *Author for correspondence: jwitte@ucsf.edu

\section{INTRODUCTION}

Over the past few years the number of samples included in genomic and other 'omic projects has exponentially increased. Such growth allows for teasing apart the complexities of polygenic disease and the effects of less common measures (e.g., rare variants) [1-3]. For example, large-scale cohort studies such as the UK Biobank now include genetic and phenotype information on 500,000 individuals [4], and other enormous cohorts are underway or planned. As another example, the number of samples included in genome-wide association studies of schizophrenia has increased more than tenfold in just 5 years via consortia efforts, which has resulted in the discovery of more than 100 novel genetic associations [5]. This trend in increasing numbers of samples is expected to continue into the foreseeable future, raising practical considerations regarding how to effectively and efficiently scale projects.

One such consideration is that of sample management, which includes the storage, transfer and tracking of samples [6]. To address storage, a common, affordable and convenient solution is the 96-well plate. This format also facilitates sample transfer through a grid-like layout. However, for large cohorts, sample transfer is both laborious and error-prone unless automated liquid-handling robots such as the Beckman Biomek are available and utilized [7,8]. As far as record-keeping goes, many research groups depend on spreadsheets (e.g., Microsoft Excel), which work in principle but quickly become unwieldy at large scale and are difficult to use for tracking the transfer of thousands of samples across various batches. Addressing the need for information technology in a laboratory setting, many Laboratory Information Management Systems have been developed, and some have achieved a comprehensive scope in capability $[9,10]$. However, these systems are growing in complexity, tending to become harder to install, configure and use. In response, some simple and easy-touse, but niche, web-based software tools have been developed for high-throughput sample processing $[11,12]$. Standing in contrast to both of these system design paradigms, Samasy was developed with the goal of being both easy-to-use and general in applicability.

As a case-in-point, we undertook a largescale re-genotyping project that required subsetting 16,600 samples from a cohort of 54,000 samples in a manner that preserved ethnicity and age balance among prostate cancer cases and controls (Emami et. al., in preparation). To address the aforementioned sample management issues, and because sample transfer was originally planned to be done by hand, we developed Samasy, a visual web-based sample management system for 96-well plates with support for automated robotic transfer. By integrating Samasy with a Beckman Biomek, we were able to reduce the number of technician hands-on hours approximately tenfold (Eunice Wan, University of California Institute for Human Genetics Genomics Core Facility, personal communication) while also decreasing the likelihood of numerous sample transfer errors (e.g., pipetting volume, well/plate mix-up, misspecified robotic well assignments, operator error, etc.). To verify the validity of Samasy's database, batch algorithm and robotic transfer file generation feature, we performed a sample transfer demonstration using six 'Source' plates, one 'Control' plate, and two 'Destination' plates with colored wells, and observed successful volume transfer with no errors (Supplementary Figure 1).

We designed Samasy to be user-friendly in manner, making installation simple, import of large datasets straight-forward, and tracking of samples visually intuitive. Samasy can visualize plate and sample data several different ways: 1) as a searchable, sortable and filterable table; 2) concep- 


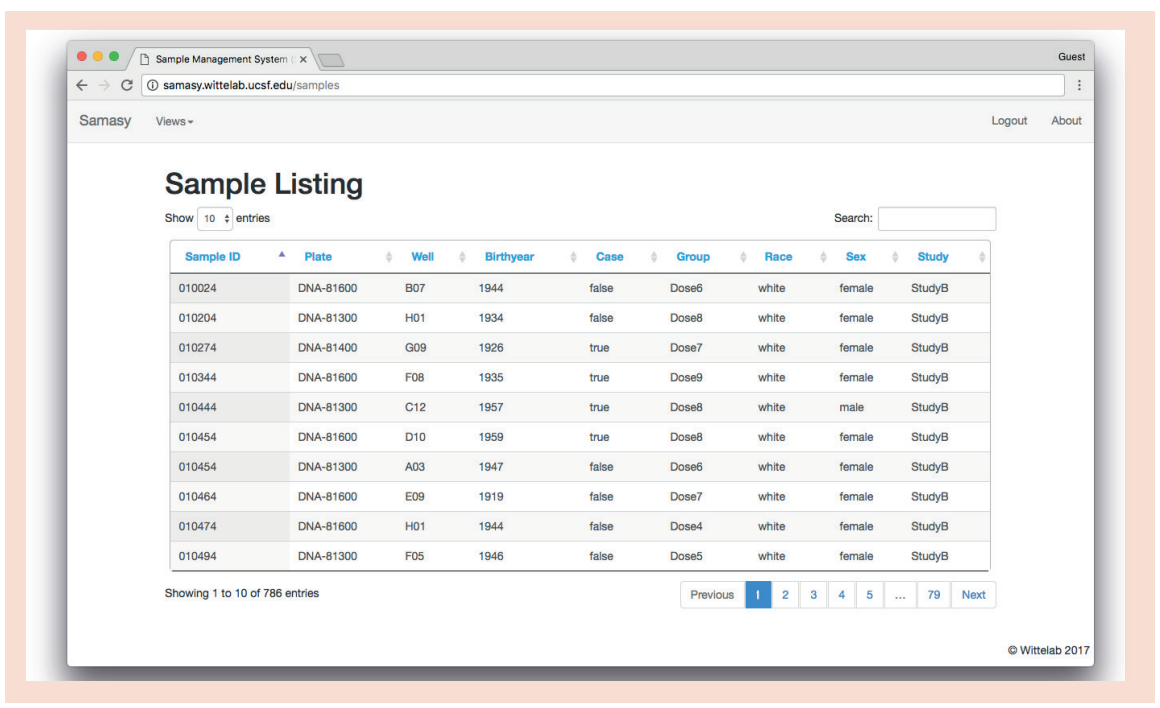

Figure 1. Samasy sample table view showing sortable and searchable sample attributes. To quickly find sample information, a search box is provided that will fuzzy match values in any attribute field. Additionally, attributes can be sorted in ascending or descending order.

- tually in a 96-well plate format with samples color coded based on user-defined attributes (e.g., age, ethnicity); 3) in batch mode, reflecting plate and sample layout on a transfer platform; or 4) as grouped attribute histograms showing sample distribution per plate or batch. Additionally, sample transfer batches can be imported, viewed and used to generate sample transfer files for automated systems (a Biomek sample transfer script is included). Samasy also provides a convenient REST API for data access, allowing integration for other uses, transfer systems and customizations, should the user find it necessary.

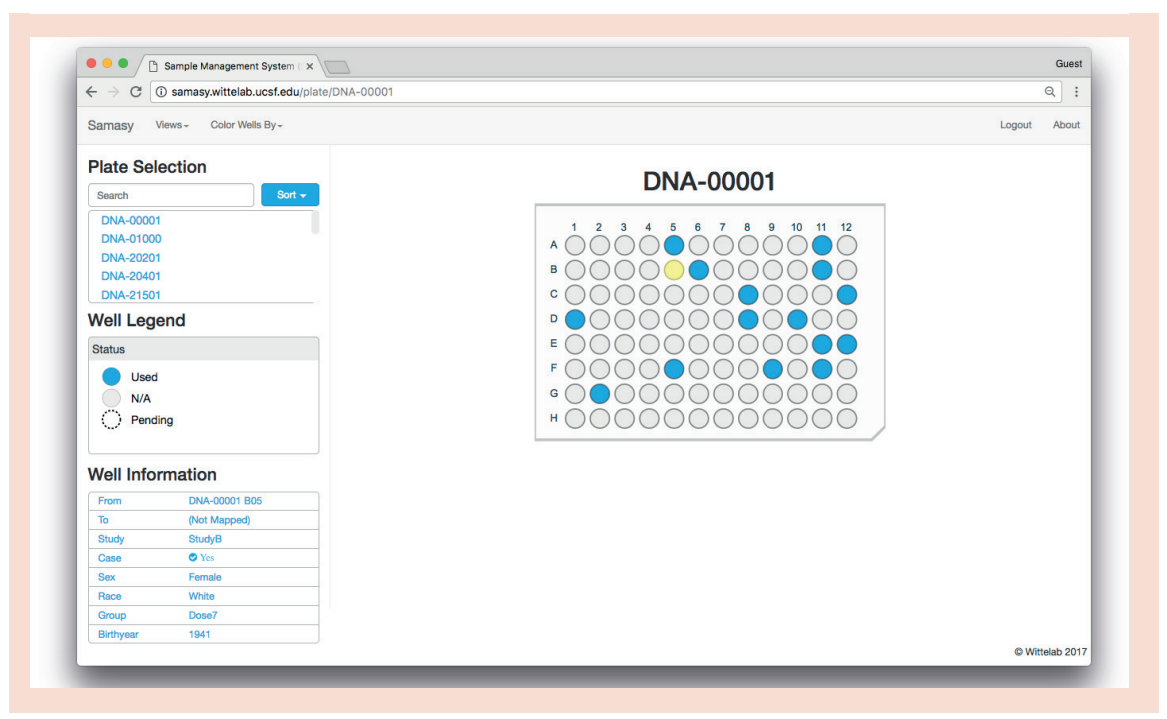

Figure 2. Plate view that shows sample locations within a 96-well plate. Wells can be clicked to access sample attribute data from the database, including to which batches they belong. Wells are color-coded by an attribute of interest using the interface menu. tions and a live demo at https://github. com/wittelab/samasy [13]), and data files are easily imported using a drag-and-drop wizard that starts automatically when the interface is first launched. The wizard will also check the integrity of each data file and provide help screens describing file formats. Afterward, the wizard creates an administration account, which allows a privileged user to import batch information and destroy data within the database. The administration user can also create, modify or destroy additional administration or unprivileged accounts.

To import data into the interface, several types of headered tab-delimited flat files (exportable by Excel) are used. The original dataset file should consist of three required columns: SampleID, PlateID and Well, and any number of user-defined sample parameters (e.g., age, ethnicity). SampleID and PlateID columns can be any alphanumeric text but the Well should be specified, such as ' $C 7$ '. The second type of flat file is the data dictionary, which defines possible sample values and maps coded data (if it exists) to corresponding real labels (e.g., 0 to 'male', 1 to 'female'). This file has three required columns: Attribute, Code and Value, where Attribute specifies the corresponding column header in the data file, Code the encoded value for a sample (e.g., 0), and Value the actual sample label (e.g., 'male'). Finally, the batch file(s), used to specify well transfer mappings can be provided. There are five required columns for this file, BatchID, Source Plate, Source Well, Destination Plate, Destination Well, and one optional column, Volume. The interface itself will produce a robot file, which is similar to a batch file but readable by sample automation platforms. For Biomek machines, the platform layout and transfer script is provided in the biomek folder. Example files are located in the example folder in the code repository. Samasy is capable of guessing the datatypes of attributes, first trying numeric types (integers or floats) before defaulting to character strings.

Once data have been imported and a user has logged in, plate, sample and batch information can be visualized. A Views drop-down menu switches between Samples (Figure 1), Plate (Figure 2), Batch (Figure 3) and Distribution (Figure 4) view modes. In the Plate and Batch views, there 
> is an additional 'Color By' drop-down menu that allows sample wells to be color-coded by sample parameters. Search and Well Legend panels are shown to the left of these two views. In the Batch view, robot files can be downloaded and batches can be marked as completed, updating sample locations in the database. Robot files can be uploaded to the Biomek machine and used to transfer samples when plates are loaded, as shown in the web interface and as specified in the Biomek transfer file (see Biomek folder). Finally, in the Distribution view, sample attributes across a plate or batch can be viewed as histograms, with the option of grouping samples by another attribute (e.g., to view the distribution of sex after grouping by ethnicity). Distributions can use ordinal or numeric data, and numeric data will be binned if a large range of values exists.

While Samasy is designed with broad use in mind, it currently only supports the 96-well-plate format and many studies store samples in denser plate formats, like the 384-well plate. Despite this limitation, many studies - including those undertaking DNA genotyping or sequencing - require volumes not amicable to these denser formats. Additionally, archival and retrospective samples are often stored in 96-well plates.

In conclusion, we have developed Samasy, a simple-to-use and intuitive web-based application to improve and optimize sample management, visualization and transfer encountered by large-scale studies utilizing 96-well plates. Starting with only a sample file and (optional) data dictionary, Samasy will generate a database to visualize plates and samples across provided parameters. Samasy will also accept sample transfer batches, provide batch views, transfer information and history, and 'robot files' to perform automated sample transfer with robotic platforms. We believe this application will serve as a useful tool for future studies requiring large-scale sample management, especially those involving genotyping or re-sequencing.

\section{FUTURE PERSPECTIVE}

Genotyping, sequencing and other 'omics projects will continue to exponentially grow in size, which will necessitate the need for robotic transfer and sample management through easy-to-use software.

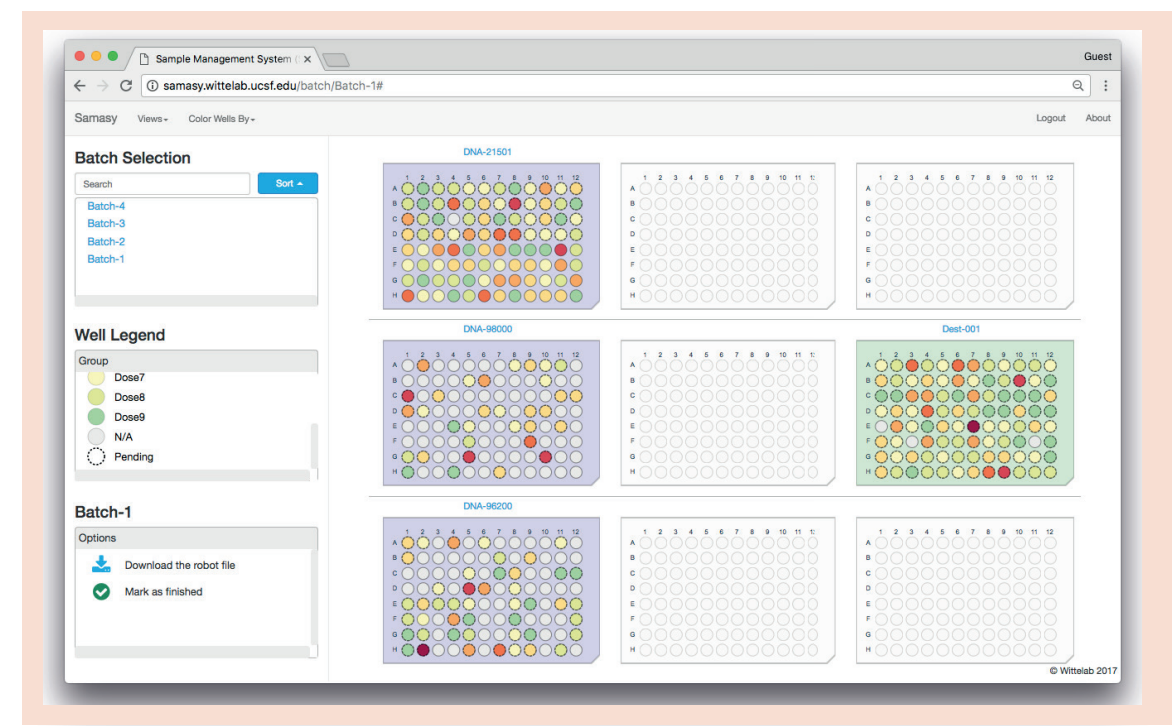

Figure 3. Samasy batch view. Plates within a batch are shown in a layout reflecting a robotic transform staging area, and plates are color coded by 'source' and 'destination' status. Wells are colored according to the attribute of interest, and well border indicates whether a sample transfer through this batch has occurred.

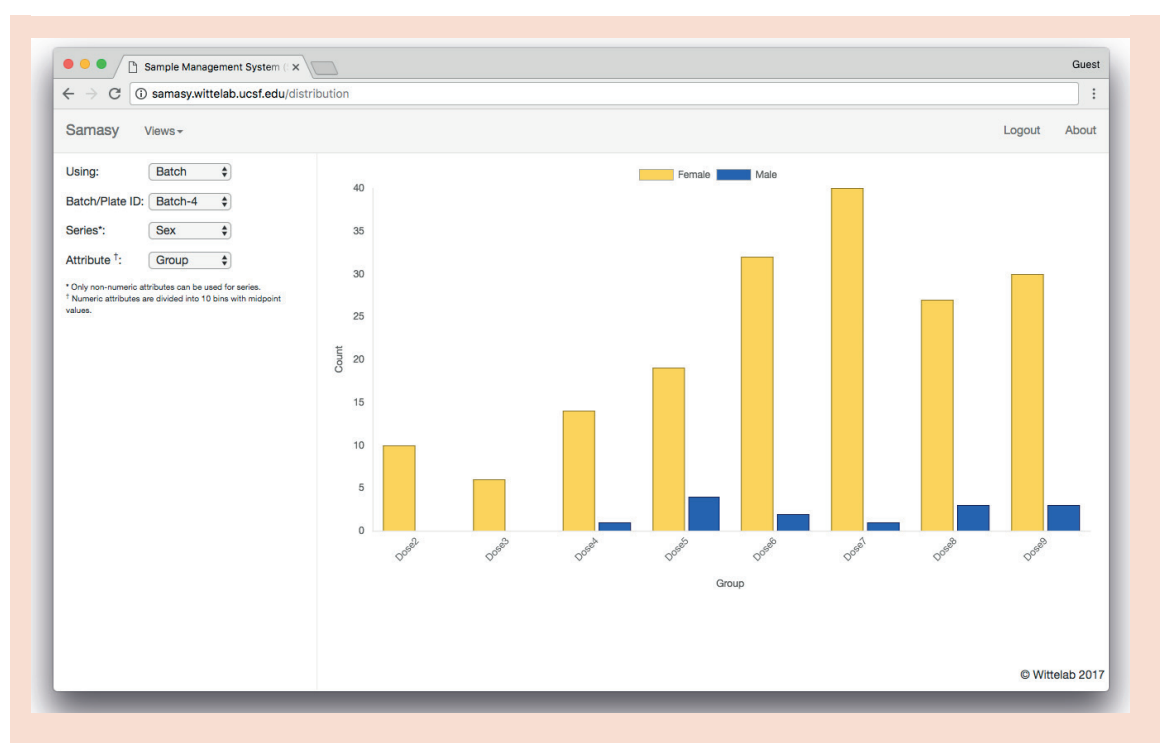

Figure 4. Samasy distribution view. Sample attribute distributions can be displayed as histograms by plate or batch. If desired, samples can also be grouped as series for distribution comparison between series class.

\section{AUTHOR CONTRIBUTIONS}

CC designed the software, performed the analysis, and wrote the paper. JW conceived the project, collected samples and wrote the paper.

\section{ACKNOWLEDGMENTS}

We would like to acknowledge the UCSF Institute of Human Genetics for their support in the testing of Samasy, Dr Pui Kwok for his helpful discussions and Eunice Wan for her feedback, discussions and testing of the Samasy interface.

\section{FINANCIAL DISCLOSURE}

This work was supported by National Institute of Health grants CA088164 and CA201358, the UCSF Goldberg-Benioff Program in Cancer Translational Biology. The authors have no other relevant affiliations or financial involvement with any organization or entity with a financial interest in or financial conflict with the subject matter or materials discussed in the 
- manuscript apart from those disclosed.

No writing assistance was utilized in the production of this manuscript.

\section{OPEN ACCESS}

This work is licensed under the AttributionNonCommercial-NoDerivatives 4.0 Unported License. To view a copy of this license, visit http://creativecommons.org/licenses/ by-nc-nd/4.0/

\section{SUPPLEMENTARY DATA}

To view the supplementary data that accompany this paper please visit the journal website at: www.future-science. com/doi/suppl/10.2144/btn-2018-0090

\section{REFERENCES}

1. Lindquist $K$, Jorgenson $E$, Hoffmann TJ, Witte JS. The impact of improved microarray coverage and larger sample sizes on future genome-wide association studies. Genet. Epidemiol. 37(4), 383-392 (2013).

2. Marouli E, Graff M, Medina-Gomez C et al. Rare and low-frequency coding variants alter human adult height. Nature 542(7640), 186-190 (2017).

3. Figueiredo JC, Stram DO, Haiman CA. The impact of GWAS findings on cancer etiology and prevention. Curr. Epidemiol. Rep. 1, 130-137 (2014).

4. UK Biobank. www.ukbiobank.ac.uk (Accessed 4 October, 2018).

5. Visscher PM, Wray NR, Zhang Q et al. 10 Years of GWAS discovery: biology, function, and translation. Am. J. Hum Genet. 101(1), 5-22 (2017).

6. Van Rossum T, Tripp B, Daley D. SLIMS - a user-friendly sample operations and inventory management system for genotyping labs. Bioinformatics 26(14), 1808-1810 (2010).

7. Andersen D, Rasmussen B, Linnet K. Validation of a fully automated robotic setup for preparation of whole blood samples for LC-MS toxicology analysis. J. Anal. Toxicol. 36(4), 280-287 (2012).

8. Kong $F$, Yuan L, Zheng $Y$, Chen W. Automatic liquid handling for life science: a critical review of the current state of the art. SLAS TECHNOLOGY: Translating Life
Sciences Innovation 17(3), 169-185 (2012).

9. Voegele C, Tavtigian SV, de Silva D, Cuber S, Thomas A, Calvez-Kelm FL. A Laboratory Information Management System (LIMS) for a high throughput genetic platform aimed at candidate gene mutation screening. Bioinformatics 23(18), 2504-2506 (2007)

10. Thurow K, Gode B, Dingerdissen U, Stoll N. Laboratory Information Management Systems for life science applcations. Organic Proc. Res. Develop. 8, 970-982 (2004).

11. Ortiz L, Pavan M, McCarthy L, Timmons J, Densmore D. automated robotic liquid handling assembly of modular DNA devices. J. Vis. Exp. 130, e54703 (2017).

12. Arvidsson $S$, Kwasniewski M, Riaño-Pachón DM, Mueller-Roeber B. QuantPrime - a flexible tool for reliable high-throughput primer design for quantitative PCR. BMC Bioinformatics 9(465), 1471-2105 (2008).

13. Samasy Github Repository. https://github.com/wittelab/samasy

(Accessed 9 October, 2018)

First draft submitted: 25 June 2018; Accepted for publication: 11 October 2018

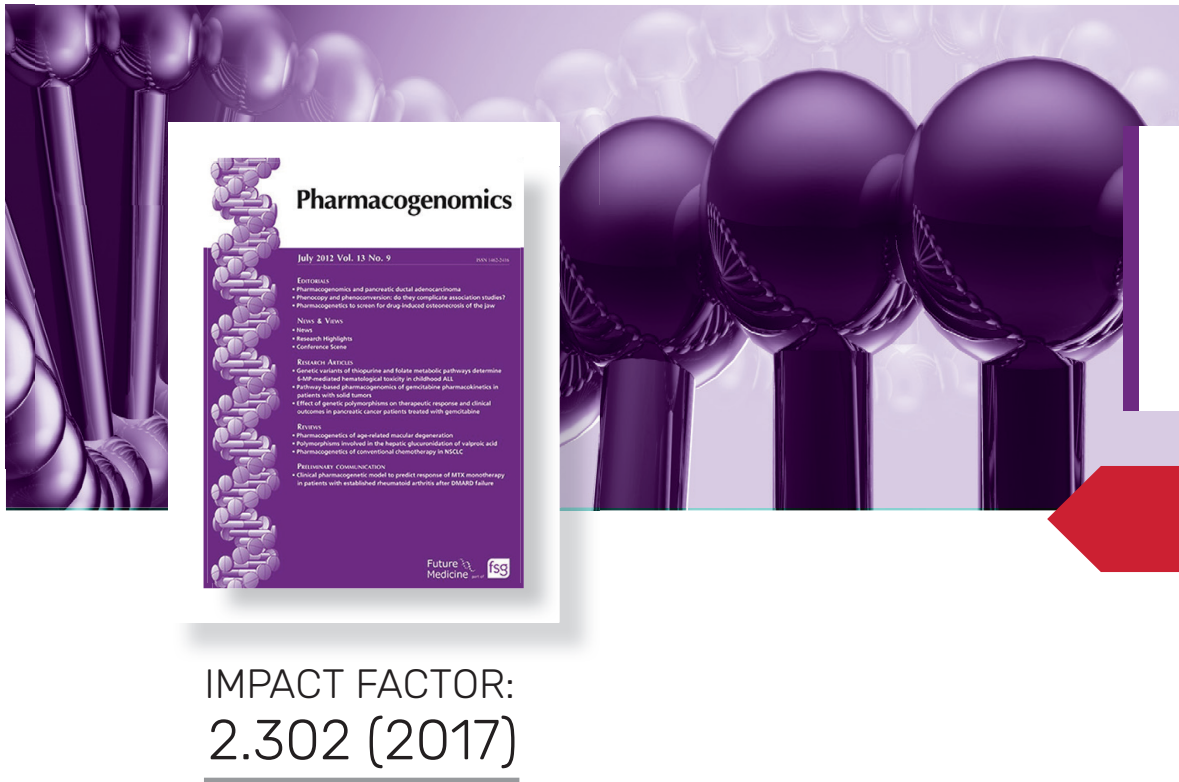

\section{Pharmacogenomics}

ISSN: 1462-2416

Frequency per year: 18

NDEXING

MEDLINE/Index Medicus,EMBASE/Excerpta Medica,

Chemical Abstracts, Science Citation Index ${ }^{\circledR}$,

Science Citation Index Expanded ${ }^{\mathrm{TM}}$ (SciSearch ${ }^{\circledR}$ ),

Biotechnology Citation Index ${ }^{\mathrm{TM}}$, Journal Citation

Reports/Science Edition ${ }^{\circledR}$, Index to Scientific Reviews ${ }^{\circledR}$,

Scopus

\section{www. futuremedicine.com}

To claim your free trial, contact us

at: trials@futuremedicine.com

quoting PGSBTN

\section{Future an imprint of Medicine}

